Laser Chem., 1999, Vol. 19, pp. 173-178

Reprints available directly from the publisher Photocopying permitted by license only
(C) 1999 OPA (Overseas Publishers Association) N.V. Published by license under the Harwood Academic Publishers imprint, part of The Gordon and Breach Publishing Group.

\title{
FEMTOSECOND TIME RESOLVED INFRARED SPECTROSCOPY OF THE ETHYLENIC STRETCH VIBRATION DURING THE ALL-TRANS TO 13-CIS ISOMERIZATION OF BACTERIORHODOPSIN
}

\author{
REINER DZIEWIOR, KARSTEN ROMEY and ROLF DILLER* \\ Institut für Experimentalphysik, Freie Universität Berlin, Arnimallee 14, \\ D-14195 Berlin, Germany
}

(Received 20 April 1997)

\begin{abstract}
We report on room temperature infrared transient absorption experiments on Bacteriorhodopsin concerning the early picosecond dynamics of the all-trans to 13-cis isomerization of the retinal chromophore. The absorption band of the all-trans chromophore ethylenic stretch vibration bleaches concomitantly to photoexcitation whereas new absorption strength is created redshifted within less than 1 ps. the initial bleach recovers partly according to the non unity quantum yield of the photoisomerization within 2 ps. With a time constant of 3 ps the low energy absorption band is downshifted $5-10 \mathrm{~cm}^{-1}$. Thus, vibrational relaxation and cooling processes appear to have ended within a few ps and on the time scale of $20 \mathrm{ps}$ a stationary BR-K difference spectrum is obtained.
\end{abstract}

Keywords: Isomerization; transient infrared spectroscopy; bacteriorhodopsin; vibrational relaxation

\section{INTRODUCTION}

The dynamics of light induced photoreactions in proteins are object of intense research. We focus here on the trans-membrane protein Bacteriorhodopsin (BR) which serves as a light driven proton pump in

\footnotetext{
* Corresponding author.
} 
Halobacteria. The pump mechanism is initiated by the all-trans to 13cis isomerization of the covalently bound retinal chromophore. In an earlier study [1] the vibrational dynamics during the first picoseconds after photoexcitation was investigated by means of subpicosecond time resolved infrared (IR) absorption spectroscopy in the spectral region of the chromophoric $\mathrm{C}=\mathrm{NH}$ stretch vibration. We have now extended this work into the region of the retinal ethylenic $(\mathrm{C}=\mathrm{C})$ stretch vibration at around $1530 \mathrm{~cm}^{-1}$.

\section{METHODS}

The pump/probe setup is based on a $1 \mathrm{kHz}$ CPM Titanium:Sapphire laser system. The pump pulses at $540 \mathrm{~nm}$ were extracted from a white light continuum, generated by the amplified pulses of about $180 \mathrm{fs}$ FWHM at $790 \mathrm{~nm}$. The infrared probe was taken from a continuous wave, liquid nitrogen cooled carbon monoxide (CO) laser, which can be tuned down to about $1450 \mathrm{~cm}^{-1}$. Time resolution was introduced by optically gating the IR probe behind the sample with a weak pulse at $790 \mathrm{~nm}$ in a $0.5 \mathrm{~mm} \mathrm{AgGaS}_{2}$-crystal. The sample was a hydrated film of light adapted $\mathrm{BR}_{570}$ (the chromophore being in all-trans configuration) between two $\mathrm{CaF}_{2}$ windows. The experiments were performed at room temperature.

\section{RESULTS AND DISCUSSION}

In Figure 1 the results are shown as a series of IR absorption transients in the region between 1536 and $1498 \mathrm{~cm}^{-1}$. Displayed is the IR absorption difference signal obtained after photoexcitation of $\mathrm{BR}_{570}$ on top of the strong background signal due to the protein amide II absorption. The strong bleach at high wavenumbers cannot be time resolved. The partial recovery of the bleach occurs within about 2 ps although a faster component, e.g., at 1526 and $1519 \mathrm{~cm}^{-1}$, respectively, contributes. The recovery turns positive at $1519 \mathrm{~cm}^{-1}$. At lower wavenumbers new absorption strength is created by the pump pulse. It rises with a time constant of about $0.7 \mathrm{ps}$ and decays partially with a time constant of not much more than 3 ps. Within the experimental 


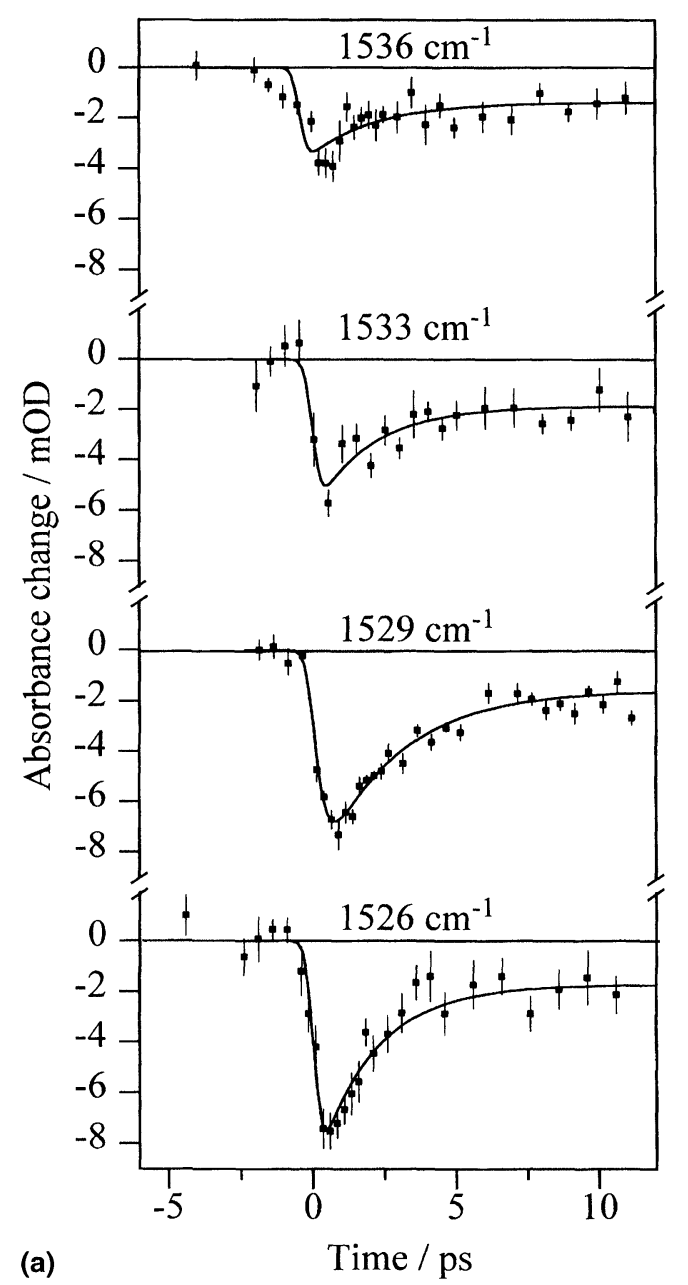

FIGURE 1a-c IR transients after photoexcitation of $\mathrm{BR}_{570}$ at $540 \mathrm{~nm}$. Data were taken at room temperature.

error of the data, the amplitudes reached after a few picoseconds remain constant in all transients on a time scale up to $20 \mathrm{ps}$ (data not shown).

According to the currently accepted picture of electronic dynamics of the BR photoisomerization obtained from optical transient absorption experiments $[2,3]$, the electronic ground state is reached 


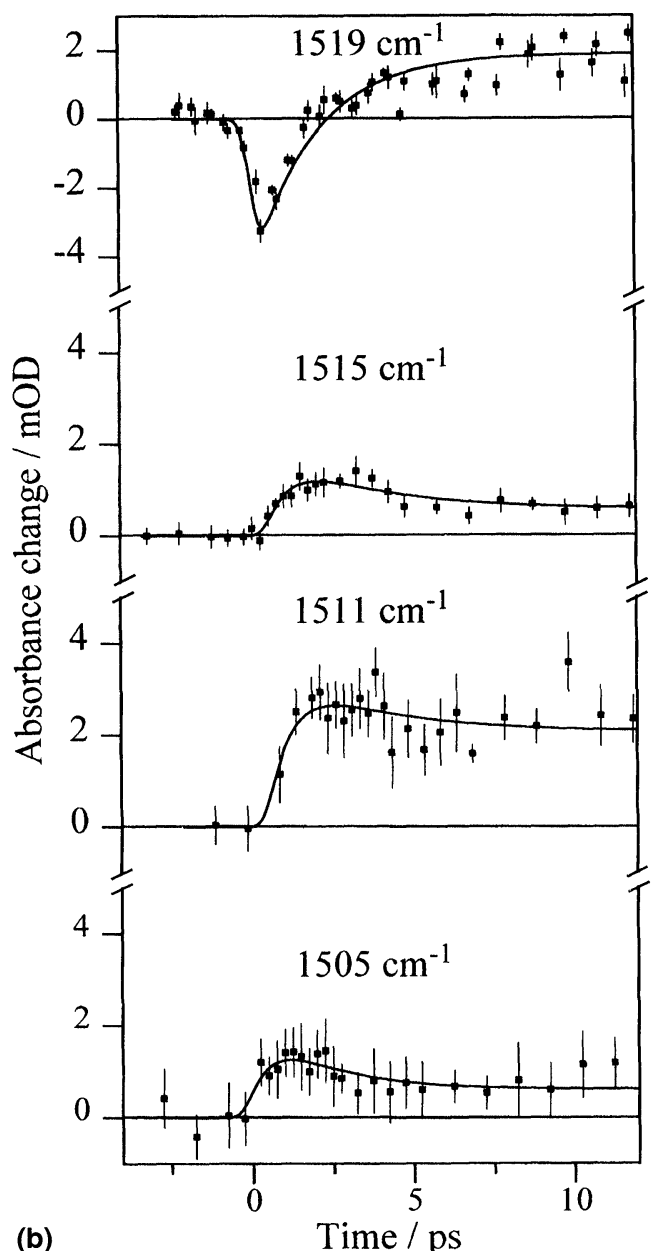

FIGURE 1 (Continued).

within $0.5-0.7 \mathrm{ps}$ as an optically red shifted intermediate J. After about 3 ps the state $K$ is formed, slightly blue shifted with respect to $J$ and stationary on the time scale of tens of picoseconds. It is still an open question as to what degree the formation of the $\mathrm{K}$ state is determined by vibrational cooling or conformational relaxation after the fast isomerization in the sterically fairly rigid protein pocket $[4,5]$. The results are interpreted as follows: Under photoexcitation the 


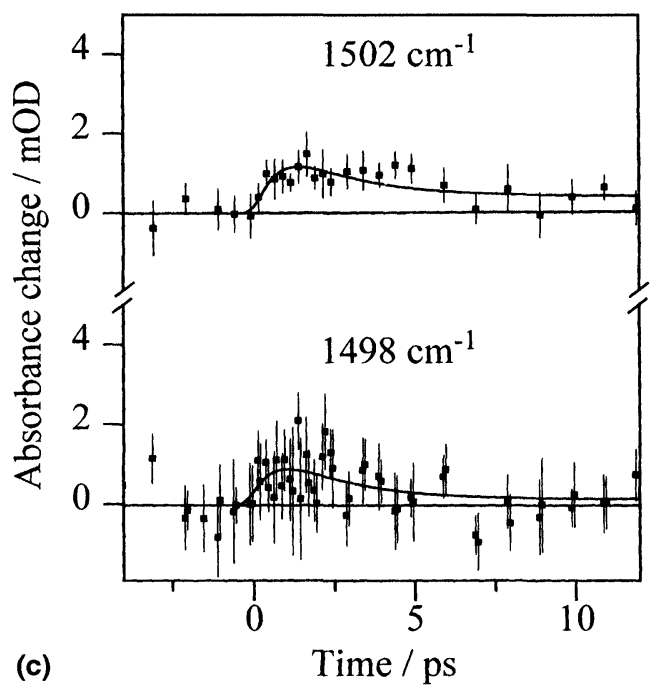

FIGURE 1 (Continued).

$\mathrm{C}=\mathrm{C}$ stretch vibration is shifted to much lower wavenumbers [6] on the time scale of the pump pulse. Thereby the bleach occurs instantaneously. According to the BR photoisomerization quantum yield of 0.6 [7], a fraction of 0.4 of the excited molecules takes the reaction path back to the $\mathrm{BR}_{570}$ ground state leading to the partial recovery at around $1529 \mathrm{~cm}^{-1}$. The slight downshift of the positive band at about $1505 \mathrm{~cm}^{-1}$ within 3 ps (cp. Fig. 2) is identified with the $J$ to $\mathrm{K}$ transition, although the recovery of $\mathrm{BR}_{570}$ as well must contribute to the optical transients. The shifting band is the signature of a vibrationally hot band but chromophoric conformational relaxation as a cause for the shift cannot be excluded without a more detailed analysis. The difference spectrum after 12 ps compares well with $\mathrm{BR}-\mathrm{K}$ difference spectra obtained by low temperature FTIR spectroscopy (e.g. [8]). As can be seen from Figure 2 the amount of recovery of the bleach is not in accordance with the fraction 0.4 , expected from the quantum yield of 0.6. Taking also into account the slight blue shift of the negative band with time and the faster component of the bleach recovery the strong bleach might be due to a stimulated emission signal in the IR. Its time course could yield information about the transition from $S_{1}$ to $S_{0}$ and the time the excited chromophore needs to become thermalized. 


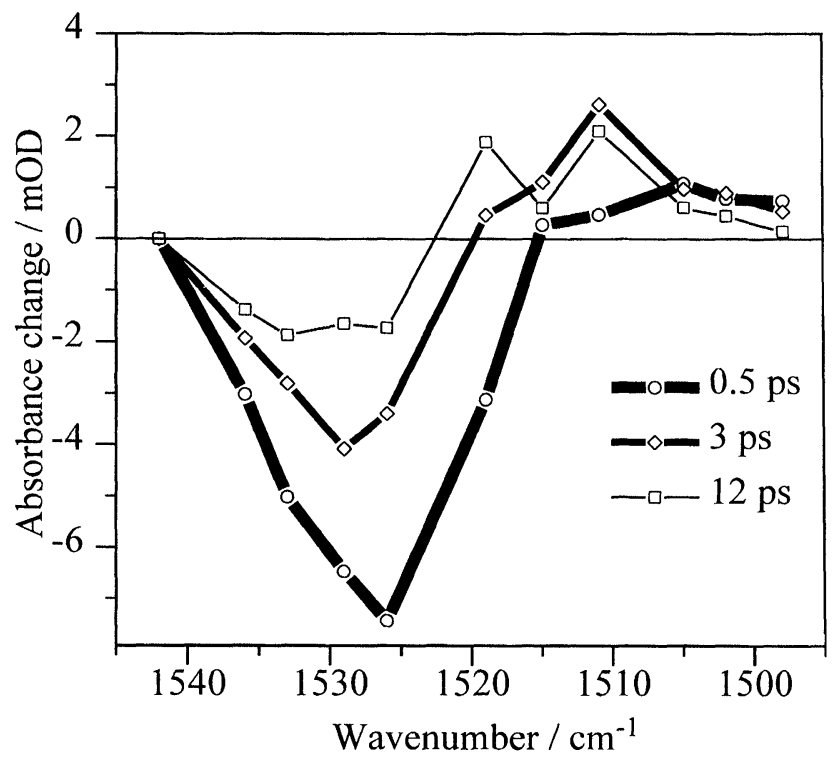

FIGURE 2 IR difference spectra, derived from the transients shown in Figure 1 at delay times of $0.5,3$ and $12 \mathrm{ps}$, respectively. The amplitudes of the data points at $1542 \mathrm{~cm}^{-1}$ are zero within the experimental error (transient data not shown).

\section{Acknowledgement}

We thank Prof. Dr. D. Stehlik for encouraging support and Prof. Dr. M. Heyn for providing the BR sample. This research was supported by the Deutsche Forschungsgemeinschaft.

\section{References}

[1] Diller, R., Maiti, S., Walker, G. C., Cowen, B. R., Pippenger, R., Bogomolni, R. A. and Hochstrasser, R. M. (1995). Chem. Phys. Lett., 241, 109.

[2] Dobler, J., Zinth, W., Kaiser, W. and Oesterhelt, D. (1988). Chem. Phys. Lett., 144, 215.

[3] Pollard, W. T., Lee, S.-Y. and Mathies, R. A. (1990). J. Chem. Phys., 92, 4012.

[4] Doig, J., Reid, P. J. and Mathies, R. A. (1991). J. Phys. Chem., 95, 6372.

[5] Brack, T. L. and Atkinson, G. H. (1991). J. Phys. Chem., 95, 2351.

[6] Kamalov, V. F., Masciangioly, T. M. and El-Sayed, M. A. (1996). J. Phys. Chem., $100,2762$.

[7] Schneider, G., Diller, R. and Stockburger, M. (1989). Chem. Phys., 131, 17.

[8] Siebert, F. and Mäntele, W. (1983). Eur. J. Biochem., 130, 565. 